\title{
Gene therapy protects heart in Friedreich's ataxia
}

A single
intravenous
dose of this
vector at
3 weeks of age
completely
protected
Mck mice
from the onset
of cardio-
myopathy

Friedreich's ataxia (FRDA) is an autosomal recessive mitochondrial disease that is characterized by neurodegeneration, diabetes and hypertrophic cardiac myopathy. The most common cause of mortality in patients with FRDA is cardiac failure, and as yet there is no treatment for FRDA-associated cardiomyopathy. Now, reporting in Nature Medicine, Perdomini and colleagues show that gene therapy can prevent the onset of cardiac disease - and even completely reverse established cardiomyopathy - in a mouse model of FRDA.

FRDA is usually caused by a trinucleotide repeat expansion in an intronic region of the frataxin

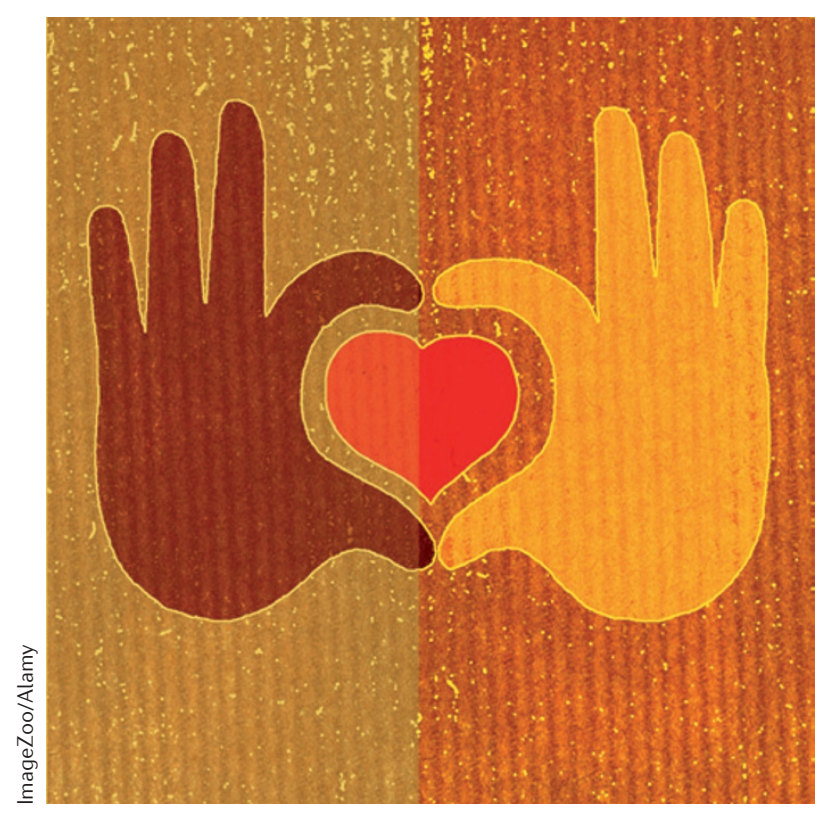

$(F X N)$ gene, resulting in reduced gene expression. FXN encodes an essential mitochondrial protein that is involved in the biosynthesis of iron-sulphur clusters, and deficiency of this protein results in mitochondrial dysfunction and mitochondrial iron overload. Mice with a conditional deletion of Fxn (Mck-Cre-Fxn ${ }^{\mathrm{L} 3 / \mathrm{L}-}$; also known as Mck mice) in cardiac and skeletal muscles develop symptoms of rapid-onset FRDA and serve as a useful mouse model of human FRDA.

To assess the potential of gene therapy for the treatment of the cardiac symptoms of FRDA, the authors selected an adeno-associated viral vector with tropism for the myocardium and dorsal root ganglia - the two tissues most affected in FRDA - and inserted a gene encoding human FXN (AAVrh10-hFXN). A single intravenous dose of this vector at 3 weeks of age completely protected Mck mice from the onset of cardiomyopathy, and molecular, cellular and physiological analysis at 35 weeks revealed no differences between cardiomyocytes of the treated Mck mice and wild-type mice. By contrast, untreated Mck mice died of cardiac failure at around 9 weeks of age.

To determine whether FXN gene therapy has any effect after the onset of heart disease, 7-week-old Mck mice with advanced cardiac insufficiency, including ventricular remodelling and left ventricular systolic dysfunction, were injected with AAVrh10-hFXN. Left ventricle function improved rapidly, reaching wild-type levels within 1-5 weeks after treatment. Moreover, a progressive normalization of the pathologyinduced transcriptional programme was observed, as well as a rapid correction of the ultrastructure of the cardiomyocytes, with complete recovery of sarcomere and mitochondria organization 5-8 weeks after treatment. Overall, the data showed a rapid biochemical, cellular and functional improvement, indicating a correction of the FRDA phenotype in cardiomyocytes. The survival of the treated $M c k$ mice was prolonged but not normal - they died prematurely owing to severe peripheral muscle atrophy. However, this was attributed to the complete lack of FXN in the skeletal muscles of the Mck mice, which contrasts with the situation in patients with FRDA, who retain some FXN expression and show no clinical symptoms related to mitochondrial impairment in skeletal muscle.

Overall, the robust correction of cardiac parameters in response to treatment with AAVrh10-hFXN, even after the onset of heart failure, suggests that gene therapy with this vector may hold promise for patients with FRDA who have severe cardiomyopathy.

Alexandra Flemming

ORIGINAL RESEARCH PAPER Perdomini, M. et al. Prevention and reversal of severe mitochondrial cardiomyopathy by gene therapy in a mouse model of Friedreich's ataxia. Nature Med. http://dx.doi.org/10.1038/nm3510 (2014) 